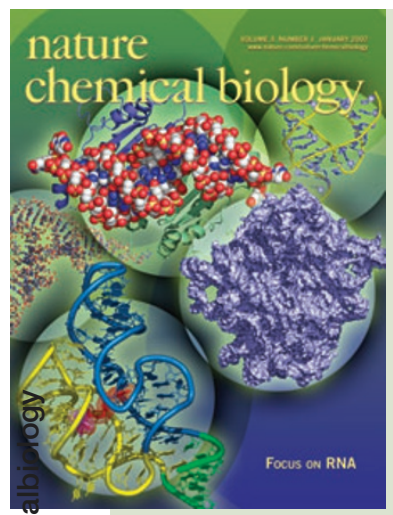

\title{
FOCUS ON RNA
}

RNA molecules occupy a central position in the flow of genetic information in all cells. Just a few decades ago, we held a relatively simple view of RNA that focused on three classes of informational RNA polymers: tRNA, mRNA and rRNA. Recent discoveries-ribozymes, RNA editing, riboswitches, RNA interference (RNAi), microRNAs and atomic insights into transcription and translation —have expanded our view of the diverse structures and functions of cellular RNA. This issue highlights the rapidly expanding frontier of RNA chemical biology.

Diverse approaches are necessary for understanding the functional roles of RNA, and the contributions from chemical biology are numerous. Ansari [Commentary, p. 2] examines the historical importance of chemical inhibitors and tools for understanding transcription and suggests how chemical and biological approaches can be combined to better understand and control informational processes in cells. This issue also highlights new structural insights that have enhanced our understanding of the ribosome [News \& Views, p. 19], the signal recognition particle, and the RNAmodifying enzyme pseudouridine synthase [Research Highlights, p. 22]. By bringing together diverse expertise, the Center for Molecular Biology of RNA at the University of California at Santa Cruz has been tackling each of these aspects of the chemistry and biology of RNA [Elements, p. 13].

The discovery of RNAi, in which small RNA molecules alter gene expression by targeting mRNAs, has added a layer of complexity to our understanding of gene regulation. In this issue, Tolia and Joshua-Tor [Review, p. 36] discuss the structure and function of Argonaute proteins and their actions as biochemical effectors of the RNAi response. Corey [Commentary, p. 8] examines the challenges facing RNAi as it moves toward becoming a tool for the clinic. He argues that knowledge from earlier studies on gene regulation by antisense and antigene oligonucleotides will be instrumental in the development of RNAi therapeutics. We also highlight a recent paper that implicates heme as a component in the biosynthetic machinery for producing microRNAs [Research Highlights, p. 22].

RNA biochemistry has always been full of surprises. Recent research has shown that cells have evolved alternate pathways to expand the repertoire of amino acids incorporated into proteins. Ambrogelly, Palioura and Söll discuss how specific tRNA modifications, tailoring enzymes and ribosomal recoding provide a biological strategy for tinkering with the genetic code [Perspective, p. 29]. We also feature two studies demonstrating that 5 '-triphosphorylated RNAs serve as a marker for sensing viral RNA in host cells [News \& Views, p. 20]. Riboswitches-mRNA motifs that regulate gene expression by specific binding to cellular metabolites - have offered another recent surprise to the RNA community. One paper demonstrates that these genetic regulatory elements may provide a new pool of therapeutic targets [Letter, p. 44; News \& Views, p. 16]. Natural and engineered RNA motifs also have broader applications. Davidson and Ellington discuss how modular RNA components can be used to create artificial and programmable genetic circuits [Perspective, p. 23]. In the coming years we should look forward to great advances in our understanding of RNA structure and function-and, perhaps, a few surprises along the way.

\section{Antibiotics that flip the riboswitch}

Riboswitches are regulatory elements within mRNA that modulate gene expression in response to metabolites. Lysine riboswitches sense lysine concentrations in order to control<smiles>[NH3+]CCS(=O)(=O)C[C@H]([NH3+])C(=O)[O-]</smiles>
subsequent lysine biosynthesis and transport. To test the idea that interfering with this riboswitch might be an effective antibacterial strategy, Blount et al. synthesized lysine analogs. The authors identified several molecules that inhibited bacterial growth by binding to the lysine riboswitch. These results suggest that rational development of small molecules that bind riboswitches may provide a new class of antibiotics. [Letters, p. 44; News \& Views, p. 16]

\section{Inteins light up possibilities}

The ability to precisely control protein expression in cells allows investigations into the biological significance of individual proteins. Schwartz et al. show that small-molecule activation of inteins to assemble a mature protein is fast, tunable and responsive to inhibitors. Furthermore, they provide the first demonstration of this splicing in a living animal, using rapamycin to initiate splicing of split luciferase in Drosophila melanogaster. [Letters, p. 50; News \& Views, p. 17]

In This Issue written by Catherine Goodman, Joanne Kotz and Terry L. Sheppard

\section{Worming into insulin signaling}

Insulin signaling is involved in regulating glucose concentrations and maintaining metabolic homeostasis. A temperaturesensitive mutant of the Caenorhabditis elegans daf-2 gene, which encodes an insulin receptor-like protein, serves as an insulin resistance model. By screening a chemical library, Min et al. identified the small molecule GAPDS as a chemical suppressor of the daf-2 phenotype. The authors found that GAPDS inhibits glyceraldehyde-3-phosphate dehydrogenase (GAPDH) by inducing the dissociation of the active tetramer into inactive monomers. GAPDH activity was found to be important in regulating the concentration of phosphoinositide, which is a known regulator of the insulin signaling pathway, thus providing mechanistic insight into this previously undescribed role of GAPDH. [Letters, p. 55] $J K$

\section{A copper switch}

To protect cells from copper-induced cell death, unused copper is sequestered by copper-binding proteins that are regulated at the level of transcription. Although a few examples of copper-sensitive transcriptional repressors are known, so far a candidate protein has not been found in most prokaryotes. Liu et al. now provide new evidence of a DNA-binding protein that is exquisitely and selectively responsive to copper with homologues in many species. Metal binding not only results in the release of the DNA for transcription, but also yields a homodimeric structure that represents a novel fold for a DNA-binding protein. [Articles, p. 60; News \& Views, p.15] 